\title{
Approximation results on Dunkl generalization of Phillips operators via $q$-calculus
}

Md. Nasiruzzaman', Aiman Mukheimer ${ }^{2}$ and M. Mursaleen ${ }^{3 *}$

"Correspondence:

mursaleenm@gmail.com

${ }^{3}$ Department of Mathematics,

Aligarh Muslim University, Aligarh,

ndia

Full list of author information is

available at the end of the article

\begin{abstract}
The main purpose of this paper is to construct $q$-Phillips operators generated by Dunkl generalization. We prove several results of Korovkin type and estimate the order of convergence in terms of several moduli of continuity.
\end{abstract}

MSC: Primary 41A25; 41A36; secondary 33C45

Keywords: Szász operator; Generating functions; Dunkl analogue; Generalization of exponential function; Modulus of continuity; Weighted modulus of continuity

\section{Introduction and auxiliary results}

In 1950, Szász [27] defined the following operators for a continuous function $f \in C[0$, $\infty)$ :

$$
S_{n}(f ; x)=e^{-n x} \sum_{k=0}^{\infty} \frac{(n x)^{k}}{k !} f\left(\frac{k}{n}\right),
$$

provided that the series is convergent. In [26], Sucu approximated the Szász-operators defined by (1.1) by Dunkl generalization with an exponential function (see [24]). For $v>-\frac{1}{2}$, Cheikh et al. [6] studied $q$-Hermite type polynomials and gave definitions of $q$-Dunkl analogues of exponential functions and recursion formula as follows:

$$
\begin{aligned}
& e_{v, q}(x)=\sum_{n=0}^{\infty} \frac{x^{n}}{\gamma_{v, q}(n)}, \quad x \in[0, \infty), \quad E_{v, q}(x)=\sum_{n=0}^{\infty} \frac{q^{\frac{n(n-1)}{2}} x^{n}}{\gamma_{v, q}(n)}, \quad x \in[0, \infty), \\
& \gamma_{v, q}(n+1)=\left(\frac{1-q^{2 v \theta_{n+1}+n+1}}{1-q}\right) \gamma_{v, q}(n), \quad n \in \mathbb{N}, \\
& \theta_{n}= \begin{cases}0 & \text { if } n \in 2 \mathbb{N}, \\
1 & \text { if } n \in 2 \mathbb{N}+1 .\end{cases}
\end{aligned}
$$

(c) The Author(s) 2019. This article is distributed under the terms of the Creative Commons Attribution 4.0 International License (http://creativecommons.org/licenses/by/4.0/), which permits unrestricted use, distribution, and reproduction in any medium, provided you give appropriate credit to the original author(s) and the source, provide a link to the Creative Commons license, and indicate if changes were made. 
The $q$-integer $[n]_{q}$ and $q$-factorial $[n]_{q}$ !, respectively, are defined by

$$
\begin{aligned}
& {[n]_{q}= \begin{cases}\frac{1-q^{n}}{1-q} & \text { for } q \neq 1, n \in \mathbb{N}, \\
1 & \text { for } q=1, \\
0 & \text { for } n=0,\end{cases} } \\
& {[n]_{q} != \begin{cases}1 & \text { for } n=0, \\
\prod_{k=1}^{n}[k]_{q} & \text { for } n \in \mathbb{N} .\end{cases} }
\end{aligned}
$$

The $q$-calculus appeared as a new area in approximation theory and has a lot of applications in different mathematical areas and physics such as number theory, combinatorics, orthogonal polynomials, basic hypergeometric functions, quantum theory, mechanics, and the theory of relativity (see [13-15]).

Içöz [11] generalized the Dunkl-Szász operators defined by (1.1) via $q$-integers as follows:

$$
D_{n, q}(f ; x)=\frac{1}{e_{v, q}\left([n]_{q} x\right)} \sum_{k=0}^{\infty} \frac{\left([n]_{q} x\right)^{k}}{\gamma_{v, q}(k)} f\left(\frac{1-q^{2 v \theta_{k}+k}}{1-q^{n}}\right),
$$

for $v>\frac{1}{2}, x \geq 0,0<q<1$ and $f \in C[0, \infty)$.

Recent improvements of Szász type operators generated by exponential function via Dunkl generalization are given in $[1-3,12,16-18,20,23,25,28]$.

The main purpose of this article is to construct the $q$-Phillips operators generated by Dunkl generalization via $q$-calculus. For more details on the approximation of classical Phillips operators via Dunkl type version, we refer to the recent article [21]. We obtain a Korovkin type result, as well as local and weighted approximations. We also study convergence properties by using the modulus of continuity and investigate the rate of convergence for functions belonging to the Lipschitz class. For further details and more information on approximation, we refer to $[9,10,19]$.

For every $f \in C_{\zeta}[0, \infty)=\left\{f \in C[0, \infty): f(t)=O\left(t^{\zeta}\right), t \rightarrow \infty\right\}$ and $x \in[0, \infty), \zeta>n, n \in$ $\mathbb{N} \cup\{0\}, v \geq-\frac{1}{2}$, we define

$$
\begin{aligned}
\mathcal{P}_{n, q}^{*}(f ; x)= & \frac{[n]_{q}}{e_{v, q}\left([n]_{q} x\right)} \sum_{k=0}^{\infty} Q_{n, q}^{v}(x) \int_{0}^{\infty / 1-q} \frac{e_{v, q}\left(-[n]_{q} t\right)[n]_{q}^{k+2 v \theta_{k}} t^{k+2 v \theta_{k}}}{\left[k+2 v \theta_{k}\right]_{q} !} \\
& \times f\left(q^{k+2 v \theta_{k}} t\right) \mathrm{d}_{q} t,
\end{aligned}
$$

where

$$
Q_{n, q}^{v}(x)=\frac{\left([n]_{q} x\right)^{k}}{\gamma_{v, q}(k)} q^{\frac{\left(k+2 v \theta_{k}\right)\left(k+2 v \theta_{k}+1\right)}{2}}
$$

For the proof of a basic estimate, we use the generalized $q$-gamma function.

Definition 1.1 The generalized $q$-gamma function is defined by

$$
\Gamma_{q}(t)=\int_{0}^{1 / 1-q} x^{t-1} E_{q}(-q x) \mathrm{d}_{q} x, \quad t>0,
$$




$$
\gamma_{q}^{A}(t)=\int_{0}^{\infty / A(1-q)} x^{t-1} e_{q}(-x) \mathrm{d}_{q} x, \quad t>0,
$$

where $\Gamma_{q}(t)=K(A ; t) \gamma_{q}^{A}(t)$ and $K(A ; t)=\frac{1}{1+A} A^{t}\left(1+\frac{1}{A}\right)_{q}^{t}(1+A)_{q}^{t-1}$. Moreover, for any positive integer $n$, we have $K(A ; n)=q^{\frac{n(n-1)}{2}}$ and $\Gamma_{q}(n)=q^{\frac{n(n-1)}{2}} \gamma_{q}^{A}(n)$, which also satisfy the following equation:

$$
\Gamma_{q}(t+1)= \begin{cases}{[t]_{q} \Gamma_{q}(t)} & \text { for } t>0 \\ 1 & \text { for } t=0\end{cases}
$$

For more details, see [8].

\section{Estimation of moments}

Lemma 2.1 Let $\mathcal{P}_{n, q}^{*}(\cdot ; \cdot)$ be the operators defined by (1.7). Then, we have

(1) $\mathcal{P}_{n, q}^{*}(1 ; x)=1$,

(2) $\mathcal{P}_{n, q}^{*}(t ; x)=x+\frac{1}{q[n]_{q}}$,

(3) $\mathcal{P}_{n, q}^{*}\left(t^{2} ; x\right) \leq \frac{(1+q)}{q^{3}[n]_{q}^{2}}+\frac{1}{q^{2}[n]_{q}}\left(1+2 q+q^{2}[1+2 v]_{q}\right) x+x^{2}$,

$$
\mathcal{P}_{n, q}^{*}\left(t^{2} ; x\right) \geq \frac{(1+q)}{q^{3}[n]_{q}^{2}}+\frac{1}{q^{2}[n]_{q}}\left(1+2 q+q^{2(1+v)}[1-2 v]_{q} \frac{e_{v, q}\left(q[n]_{q} x\right)}{e_{v, q}\left([n]_{q} x\right)}\right) x+x^{2},
$$

(4) $\quad \mathcal{P}_{n, q}^{*}\left(t^{3} ; x\right) \leq \frac{(1+q)\left(1+q+q^{2}\right)}{q^{6}[n]_{q}^{3}}$

$$
\begin{aligned}
& +\frac{1}{q^{5}[n]_{q}^{2}}\left\{\left(1+3 q+4 q^{2}+3 q^{3}\right)+q^{2}\left(1+2 q+3 q^{2}\right)[1+2 v]_{q}\right. \\
& \left.+q^{5}[1+2 v]_{q}^{2}\right\} x \\
& +\frac{1}{q^{4}[n]_{q}}\left\{q\left(1+2 q+3 q^{2}\right)+3 q^{4}[1+2 v]_{q}\right\} x^{2}+x^{3},
\end{aligned}
$$

(5) $\mathcal{P}_{n, q}^{*}\left(t^{4} ; x\right) \leq \frac{(1+q)\left(1+2 q+3 q^{2}+3 q^{3}+2 q^{4}+q^{5}\right)}{q^{10}[n]_{q}^{4}}$

$$
\begin{aligned}
& +\frac{1}{q^{9}[n]_{q}^{3}}\left\{\left(1+4 q+8 q^{2}+12 q^{3}+12 q^{4}+9 q^{5}+4 q^{6}\right)\right. \\
& +q^{2}\left(1+3 q+7 q^{2}+9 q^{3}+9 q^{4}+6 q^{5}\right)[1+2 v]_{q} \\
& \left.+q^{5}\left(1+2 q+3 q^{2}+4 q^{3}\right)[1+2 v]_{q}^{2}+q^{9}[1+2 v]_{q}^{3}\right\} x \\
& +\frac{1}{q^{8}[n]_{q}^{2}}\left\{q\left(1+3 q+7 q^{2}+9 q^{3}+9 q^{4}+6 q^{5}\right)\right. \\
& \left.+q^{4}\left(1+2 q+3 q^{2}+4 q^{3}\right)[1+2 v]_{q}+7 q^{8}[1+2 v]_{q}^{2}\right\} x^{2} \\
& +\frac{1}{q^{7}[n]_{q}}\left\{q^{3}\left(1+2 q+3 q^{2}+4 q^{3}\right)+6 q^{7}[1+2 v]_{q}\right\} x^{3}+x^{4} .
\end{aligned}
$$


Proof We prove this lemma by using the definition of generalized $q$-gamma function defined by Definition 1.1. More precisely,

$$
\begin{aligned}
\int_{0}^{\infty / 1-q} q^{\frac{\left(k+2 v \theta_{k}\right)\left(k+2 v \theta_{k}+1\right)}{2}} \frac{e_{v, q}\left(-[n]_{q} t\right)[n]_{q}^{k+2 v \theta_{k}} t^{k+2 v \theta_{k}}}{\left[k+2 v \theta_{k}\right]_{q} !}\left(q^{k+2 v \theta_{k}} t\right)^{u} \mathrm{~d}_{q} t \\
=\frac{1}{[n]_{q}^{u+1}} \frac{1}{\left[k+2 v \theta_{k}\right]_{q} !} q^{\frac{\left(k+2 v \theta_{k}\right)\left(k+2 v \theta_{k}+1\right)}{2}+u\left(k+2 v \theta_{k}\right)} \\
\quad \times \int_{0}^{\infty / 1-q}\left([n]_{q} t\right)^{k+2 v \theta_{k}+u} e_{v, q}\left(-[n]_{q} t\right)[n]_{q} \mathrm{~d}_{q} t \\
=\frac{1}{[n]_{q}^{u+1}} \frac{1}{\left[k+2 v \theta_{k}\right]_{q} q^{\frac{\left(k+2 v \theta_{k}\right)\left(k+2 v \theta_{k}+1\right)}{2}}+u\left(k+2 v \theta_{k}\right)} \int_{0}^{\infty / 1-q} t^{k+2 v \theta_{k}+u} e_{v, q}(-t) \mathrm{d}_{q} t \\
=\frac{1}{[n]_{q}^{u+1}} \frac{1}{\left[k+2 v \theta_{k}\right]_{q} !} q^{\frac{\left(k+2 v \theta_{k}\right)\left(k+2 v \theta_{k}+1\right)}{2}+u\left(k+2 v \theta_{k}\right)} \gamma_{q}^{1}\left(k+2 v \theta_{k}+u+1\right) \\
=\frac{1}{[n]_{q}^{u+1}} \frac{1}{\left[k+2 v \theta_{k}\right]_{q} !} q^{\frac{\left(k+2 v \theta_{k}\right)\left(k+2 v \theta_{k}+1\right)}{2}+u\left(k+2 v \theta_{k}\right)} \frac{\left[k+2 v \theta_{k}+u\right]_{q} !}{q^{\frac{\left(k+2 v \theta_{k}+u\right)\left(k+2 v \theta_{k}+u+1\right)}{2}}} \\
=\frac{1}{[n]_{q}^{u+1}} \frac{\left[k+2 v \theta_{k}+u\right]_{q} !}{\left[k+2 v \theta_{k}\right]_{q} !} \frac{1}{q^{\frac{u(u+1)}{2}}} .
\end{aligned}
$$

If $u=0$, then $f(t)=1$, and hence

$$
\begin{aligned}
\mathcal{P}_{n, q}^{*}(1 ; x) & =\frac{[n]_{q}}{e_{v, q}\left([n]_{q} x\right)} \sum_{k=0}^{\infty} \frac{\left([n]_{q} x\right)^{k}}{\gamma_{v, q}(k)} \frac{\left[k+2 v \theta_{k}\right]_{q} !}{[n]_{q}\left[k+2 v \theta_{k}\right]_{q} !} \\
& =1 .
\end{aligned}
$$

If $u=1$, then $f(t)=t$, and hence

$$
\begin{aligned}
\mathcal{P}_{n, q}^{*}(t ; x)= & \frac{[n]_{q}}{e_{v, q}\left([n]_{q} x\right)} \sum_{k=0}^{\infty} \frac{\left([n]_{q} x\right)^{k}}{\gamma_{v, q}(k)} \frac{\left[k+2 v \theta_{k}+1\right]_{q} !}{q[n]_{q}^{2}\left[k+2 v \theta_{k}\right]_{q} !} \\
= & \frac{1}{q[n]_{q} e_{v, q}\left([n]_{q} x\right)} \sum_{k=0}^{\infty} \frac{\left([n]_{q} x\right)^{k}}{\gamma_{v, q}(k)}\left[k+2 v \theta_{k}+1\right]_{q} \\
= & \frac{1}{q[n]_{q} e_{v, q}\left([n]_{q} x\right)} \sum_{k=0}^{\infty} \frac{\left([n]_{q} x\right)^{k}}{\gamma_{v, q}(k)} \\
& +\frac{1}{[n]_{q} e_{v, q}\left([n]_{q} x\right)} \sum_{k=0}^{\infty} \frac{\left([n]_{q} x\right)^{k}}{\gamma_{v, q}(k)}\left[k+2 v \theta_{k}\right]_{q} \\
= & x+\frac{1}{q[n]_{q}} .
\end{aligned}
$$

Take $u=2$, then, for $f(t)=t^{2}$, we have

$$
\begin{aligned}
\mathcal{P}_{n, q}^{*}\left(t^{2} ; x\right) & =\frac{[n]_{q}}{e_{v, q}\left([n]_{q} x\right)} \sum_{k=0}^{\infty} \frac{\left([n]_{q} x\right)^{k}}{\gamma_{v, q}(k)} \frac{\left[k+2 v \theta_{k}+2\right]_{q} !}{q^{3}[n]_{q}^{3}\left[k+2 v \theta_{k}\right]_{q} !} \\
& =\frac{1}{q^{3}[n]_{q}^{2} e_{v, q}\left([n]_{q} x\right)} \sum_{k=0}^{\infty} \frac{\left([n]_{q} x\right)^{k}}{\gamma_{v, q}(k)}\left[k+2 v \theta_{k}+2\right]_{q}\left[k+2 v \theta_{k}+1\right]_{q}
\end{aligned}
$$




$$
\begin{aligned}
= & \frac{1}{q^{3}[n]_{q}^{2} e_{v, q}\left([n]_{q} x\right)} \\
& \times \sum_{k=0}^{\infty} \frac{\left([n]_{q} x\right)^{k}}{\gamma_{v, q}(k)}\left\{(1+q)+q(1+2 q)\left[k+2 v \theta_{k}\right]_{q}+q^{3}\left[k+2 v \theta_{k}\right]_{q}^{2}\right\} \\
= & \frac{(1+q)}{q^{3}[n]_{q}^{2}}+\frac{(1+2 q)}{q^{2}[n]_{q}} x+\frac{1}{[n]_{q}^{2} e_{v, q}\left([n]_{q} x\right)} \sum_{k=0}^{\infty} \frac{\left([n]_{q} x\right)^{k}}{\gamma_{v, q}(k)}\left[k+2 v \theta_{k}\right]_{q}^{2} .
\end{aligned}
$$

From [11] and by (1.6), we obtain

$$
\begin{aligned}
{[n]_{q}^{2} x^{2}+q^{2 v}[1-2 v]_{q} \frac{e_{v, q}\left(q[n]_{q} x\right)}{e_{v, q}\left([n]_{q} x\right)}[n]_{q} x } & \leq \frac{1}{[n]_{q}^{2} e_{v, q}\left([n]_{q} x\right)} \sum_{k=0}^{\infty} \frac{\left([n]_{q} x\right)^{k}}{\gamma_{v, q}(k)} \\
& \leq[n]_{q}^{2} x^{2}+[1+2 v]_{q}[n]_{q} x .
\end{aligned}
$$

For $u=3, f(t)=t^{3}$ and for $u=4, f(t)=t^{4}$, we get

$$
\mathcal{P}_{n, q}^{*}\left(t^{3} ; x\right)=\frac{1}{q^{4}[n]_{q}^{3} e_{v, q}\left([n]_{q} x\right)} \sum_{k=0}^{\infty} \frac{\left([n]_{q} x\right)^{k}}{\gamma_{v, q}(k)}\left[k+2 v \theta_{k}+3\right]_{q}\left[k+2 v \theta_{k}+2\right]_{q}\left[k+2 v \theta_{k}+1\right]_{q}
$$

and

$$
\begin{aligned}
\mathcal{P}_{n, q}^{*}\left(t^{4} ; x\right)= & \frac{1}{q^{10}[n]_{q}^{4} e_{v, q}\left([n]_{q} x\right)} \sum_{k=0}^{\infty} \frac{\left([n]_{q} x\right)^{k}}{\gamma_{v, q}(k)} \\
& \times\left[k+2 v \theta_{k}+4\right]_{q}\left[k+2 v \theta_{k}+3\right]_{q}\left[k+2 v \theta_{k}+2\right]_{q}\left[k+2 v \theta_{k}+1\right]_{q} .
\end{aligned}
$$

A simple calculation leads to

$$
\begin{aligned}
{[k+} & \left.2 v \theta_{k}+3\right]_{q}\left[k+2 v \theta_{k}+2\right]_{q}\left[k+2 v \theta_{k}+1\right]_{q} \\
= & (1+q)\left(1+q+q^{2}\right)+\left\{q(1+2 q)\left(1+q+q^{2}\right)+q^{3}(1+q)\right\}\left[k+2 v \theta_{k}\right]_{q} \\
& +\left\{q^{3}\left(1+q+q^{2}\right)+q^{4}(1+2 q)\right\}\left[k+2 v \theta_{k}\right]_{q}^{2}+q^{6}\left[k+2 v \theta_{k}\right]_{q}^{3}, \\
{[k+} & \left.2 v \theta_{k}+4\right]_{q}\left[k+2 v \theta_{k}+3\right]_{q}\left[k+2 v \theta_{k}+2\right]_{q}\left[k+2 v \theta_{k}+1\right]_{q} \\
= & (1+q)\left(1+2 q+3 q^{2}+3 q^{3}+2 q^{4}+q^{5}\right)+\left\{q(1+2 q)\left(1+2 q+3 q^{2}+3 q^{3}+2 q^{4}+q^{5}\right)\right. \\
& \left.+q^{3}(1+q)\left(1+2 q+2 q^{2}+2 q^{3}\right)\right\}\left[k+2 v \theta_{k}\right]_{q} \\
& +\left\{q^{3}\left(1+2 q+3 q^{2}+3 q^{3}+2 q^{4}+q^{5}\right)\right. \\
& \left.+q^{4}(1+2 q)\left(1+2 q+2 q^{2}+2 q^{3}\right)+q^{7}(1+q)\right\}\left[k+2 v \theta_{k}\right]_{q}^{2} \\
& +\left\{q^{6}\left(1+2 q+2 q^{2}+2 q^{3}\right)+q^{8}(1+2 q)\right\}\left[k+2 v \theta_{k}\right]_{q}^{3}+q^{10}\left[k+2 v \theta_{k}\right]_{q}^{4} .
\end{aligned}
$$

Hence by using the result for $D_{n, q}(f ; x)$ defined by $(1.6)$ with $f(t)=t^{3}$ and $f(t)=t^{4}$ (see [11]), we get the required result.

Lemma 2.2 Let $\mathcal{P}_{n, q}^{*}(\cdot ; \cdot)$ be the operators defined by (1.7). Then, we have

$1^{\circ} \quad \mathcal{P}_{n, q}^{*}((t-x) ; x)=\frac{1}{q[n]_{q}}$, 


$$
\begin{aligned}
2^{\circ} \quad \mathcal{P}_{n, q}^{*}\left((t-x)^{2} ; x\right) & \leq \frac{(1+q)}{q^{3}[n]_{q}^{2}}+\frac{1}{q^{2}[n]_{q}}\left(1+q^{2}[1+2 v]_{q}\right) x, \\
\mathcal{P}_{n, q}^{*}\left((t-x)^{2} ; x\right) & \geq \frac{(1+q)}{q^{3}[n]_{q}^{2}}+\frac{1}{q^{2}[n]_{q}}\left(1+q^{2(1+v)}[1-2 v]_{q} \frac{e_{v, q}\left(q[n]_{q} x\right)}{e_{v, q}\left([n]_{q} x\right)}\right) x .
\end{aligned}
$$

\section{Korovkin and weighted Korovkin type approximation}

Korovkin's approximation theory [4] has many applications in classical approximation theory, as well as in other branches of mathematics. In this section we obtained some approximation results via well known Korovkin's type theorem and weighted Korovkin's type theorem for the operators defined by (1.7).

Let $C_{B}\left(\mathbb{R}^{+}\right)$be the set of all bounded and continuous functions on $\mathbb{R}^{+}=[0, \infty)$, which is a linear normed space with

$$
\|f\|_{C_{B}}=\sup _{x \geq 0}|f(x)|
$$

Let

$$
E:=\left\{f: x \in[0, \infty), \frac{f(x)}{1+x^{2}} \text { is convergent as } x \rightarrow \infty\right\} .
$$

In order to obtain the convergence results for the operators $\mathcal{P}_{n, q}^{*}(\cdot ; \cdot)$ defined by $(1.7)$, we take $q=q_{n}\left(0<q_{n}<1\right)$ such that

$$
\lim _{n \rightarrow \infty} q_{n}=1 \quad \text { and } \quad \lim _{n \rightarrow \infty} q_{n}^{n}=\alpha
$$

for some constant $\alpha(0 \leqq \alpha<1)$.

Theorem 3.1 Let $q=q_{n}$, with $0<q_{n}<1$, satisfy (3.1). Then, for any function $f \in C[0$, $\infty) \cap E$,

$$
\lim _{n \rightarrow \infty} \mathcal{P}_{n, q_{n}}^{*}(f ; x)=f(x)
$$

Proof The proof is based on the well-known Korovkin's theorem regarding the convergence of a sequence of linear and positive operators, so it is enough to prove the conditions

$$
\lim _{n \rightarrow \infty} \mathcal{P}_{n, q_{n}}^{*}\left(t^{j} ; x\right)=x^{j}, \quad j=0,1,2,
$$

uniformly on $[0,1]$.

Clearly, $\frac{1}{[n]_{q}} \rightarrow 0,(n \rightarrow \infty)$ we have

$$
\lim _{n \rightarrow \infty} \mathcal{P}_{n, q_{n}}^{*}(t ; x)=x, \quad \lim _{n \rightarrow \infty} \mathcal{P}_{n, q_{n}}^{*}\left(t^{2} ; x\right)=x^{2} .
$$

This completes the proof.

We recall the weighted spaces of functions on $\mathbb{R}^{+}$, which are defined as follows:

$$
P_{\sigma}\left(\mathbb{R}^{+}\right)=\left\{f:|f(x)| \leq M_{f} \sigma(x)\right\}
$$




$$
\begin{aligned}
& Q_{\sigma}\left(\mathbb{R}^{+}\right)=\left\{f: f \in P_{\sigma}\left(\mathbb{R}^{+}\right) \cap C[0, \infty)\right\}, \\
& Q_{\sigma}^{k}\left(\mathbb{R}^{+}\right)=\left\{f: f \in Q_{\sigma}\left(\mathbb{R}^{+}\right) \text {and } \lim _{x \rightarrow \infty} \frac{f(x)}{\sigma(x)}=k(k \text { is a constant })\right\},
\end{aligned}
$$

where $\sigma(x)=1+x^{2}$ is a weight function and $M_{f}$ is a constant depending only on $f$. Note that $Q_{\sigma}\left(\mathbb{R}^{+}\right)$is a normed space with the norm $\|f\|_{\sigma}=\sup _{x \geq 0} \frac{|f(x)|}{\sigma(x)}$.

Theorem 3.2 Let $q=q_{n}$, with $0<q_{n}<1$, satisfy (3.1). Then, for any function $f \in Q_{\sigma}^{k}\left(\mathbb{R}^{+}\right)$, we have

$$
\lim _{n \rightarrow \infty}\left\|\mathcal{P}_{n, q_{n}}^{*}(f ; \cdot)-f\right\|_{\sigma}=0
$$

Proof Take $f(t)=t^{\tau}$. Then since $f(t) \in C_{\sigma}^{k}\left(\mathbb{R}^{+}\right)$, by Korovkin's theorem, it satisfies $\mathcal{P}_{n, q_{n}}^{*}\left(t^{\tau}\right.$; $x) \rightarrow x^{\tau}$ uniformly, whenever $n \rightarrow \infty$. Therefore, by applying Lemma 2.1, since $\mathcal{P}_{n, q_{n}}^{*}(1$; $x)=1$, we have

$$
\lim _{n \rightarrow \infty}\left\|\mathcal{P}_{n, q_{n}}^{*}(1 ; \cdot)-1\right\|_{\sigma}=0
$$

and

$$
\begin{aligned}
\left\|\mathcal{P}_{n, q_{n}}^{*}(t ; x)-\cdot\right\|_{\sigma} & =\sup _{x \in[0, \infty)} \frac{\left|\mathcal{P}_{n, q_{n}}^{*}(t ; x)-x\right|}{1+x^{2}} \\
& =\frac{1}{\left.q_{n}[n]\right]_{q_{n}}} \sup _{x \in[0, \infty)} \frac{1}{1+x^{2}} .
\end{aligned}
$$

Then, clearly, $\frac{1}{[n]_{q_{n}}} \rightarrow 0$ as $n \rightarrow \infty$, which implies that

$$
\lim _{n \rightarrow \infty}\left\|\mathcal{P}_{n, q_{n}}^{*}(t ; \cdot)-x\right\|_{\sigma}=0
$$

In similar way,

$$
\begin{aligned}
\left\|\mathcal{P}_{n, q_{n}}^{*}\left(t^{2} ; \cdot\right)-x^{2}\right\|_{\sigma}= & \sup _{x \in[0, \infty)} \frac{\left|\mathcal{P}_{n, q_{n}}^{*}\left(t^{2} ; x\right)-x^{2}\right|}{1+x^{2}} \\
= & \frac{1}{q_{n}^{2}[n]_{q_{n}}}\left(1+2 q_{n}+q_{n}^{2}[1+2 v]_{q_{n}}\right) \sup _{x \in[0, \infty)} \frac{x}{1+x^{2}} \\
& +\frac{\left(1+q_{n}\right)}{q_{n}^{3}[n]_{q_{n}}^{2}} \sup _{x \in[0, \infty)} \frac{1}{1+x^{2}} .
\end{aligned}
$$

Thus we have

$$
\lim _{n \rightarrow \infty}\left\|\mathcal{P}_{n, q_{n}}^{*}\left(t^{2} ; \cdot\right)-x^{2}\right\|_{\sigma}=0 .
$$

This completes the proof.

\section{Order of approximation}

The modulus of continuity of $f$ denoted by $\omega(f ; \delta)$ gives the maximum oscillation of $f$ in any interval of length not exceeding $\delta>0$. For a function $f \in C_{B}\left(\mathbb{R}^{+}\right)$, it is given 
by

$$
\omega(f ; \delta)=\sup _{|t-x| \leq \delta}|f(t)-f(x)| ; \quad t, x \in[0, \infty)
$$

and, for any $\delta>0$, one has

$$
|f(t)-f(x)| \leq\left(\frac{|t-x|}{\delta}+1\right) \omega(f ; \delta)
$$

Theorem 4.1 Let $f \in C_{B}\left(\mathbb{R}^{+}\right)$and $x \in[0, \infty)$. Then we have

$$
\begin{aligned}
& \left|\mathcal{P}_{n, q_{n}}^{*}(f ; x)-f(x)\right| \\
& \quad \leq\left\{1+\sqrt{\frac{\left(1+q_{n}\right)}{q_{n}^{3}[n]_{q_{n}}}+\frac{1}{q_{n}^{2}}\left(1+q_{n}^{2}[1+2 v]_{q_{n}}\right) x}\right\} \omega\left(f ; \frac{1}{\sqrt{[n]_{q_{n}}}}\right),
\end{aligned}
$$

where $q=q_{n}$ are numbers such that $0<q_{n}<1$ and (3.1) holds, and $\omega(f ; \cdot)$ is the modulus of continuity defined by (4.1).

Proof We prove it by using (4.1)-(4.2) and Cauchy-Schwarz inequality. Indeed,

$$
\begin{aligned}
&\left|\mathcal{P}_{n, q_{n}}^{*}(f ; x)-f(x)\right| \leq \frac{[n]_{q_{n}}}{e_{v, q_{n}}\left([n]_{q_{n}} x\right)} \sum_{k=0}^{\infty} Q_{n, q_{n}}^{v}(x) \int_{0}^{\infty / 1-q_{n}} \frac{e_{v, q_{n}}\left(-[n]_{q_{n}} t\right)[n]_{q_{n}}^{k+2 v \theta_{k}} t^{k+2 v \theta_{k}}}{\left[k+2 v \theta_{k}\right]_{q_{n}} !} \\
& \times\left|f\left(q_{n}^{k+2 v \theta_{k}} t\right)-f(x)\right| \mathrm{d}_{q_{n}} t \\
& \leq \frac{[n]_{q_{n}}}{e_{v, q_{n}}\left([n]_{q_{n}} x\right)} \sum_{k=0}^{\infty} Q_{n, q_{n}}^{v}(x) \\
& \times \int_{0}^{\infty / 1-q_{n}} \frac{e_{v, q_{n}}\left(-[n]_{q_{n}} t\right)[n]_{q_{n}}^{k+2 v \theta_{k}} t^{k+2 v \theta_{k}}}{\left[k+2 v \theta_{k}\right]_{q_{n}} !}\left(1+\frac{1}{\delta}\left|q_{n}^{k+2 v \theta_{k}} t-x\right|\right) \mathrm{d}_{q_{n}} t \omega(f ; \delta) \\
&=\left\{\frac { 1 } { \delta } \left(\frac{[n]_{q_{n}}}{e_{v, q_{n}}\left([n]_{q_{n}} x\right)} \sum_{k=0}^{\infty} Q_{n, q_{n}}^{v}(x)\right.\right. \\
&\left.\left.\times \int_{0}^{\infty / 1-q_{n}} \frac{e_{v, q_{n}}\left(-[n]_{q_{n}} t\right)[n]_{q_{n}}^{k+2 v \theta_{k}} t^{k+2 v \theta_{k}}}{\left[k+2 v \theta_{k}\right]_{q_{n}} !}\left(\left|q_{n}^{k+2 v \theta_{k}} t-x\right|\right) \mathrm{d}_{q_{n}} t\right)+1\right\} \omega(f ; \delta) \\
& \leq\left\{1+\frac{1}{\delta} \frac{[n]_{q_{n}}}{e_{v, q_{n}}\left([n]_{q_{n}} x\right)} \sum_{k=0}^{\infty} Q_{n, q_{n}}^{v}(x)\right. \\
&\left.\times \int_{0}^{\infty / 1-q_{n}} \frac{e_{v, q_{n}}\left(-[n]_{q_{n}} t\right)[n]_{q_{n}}^{k+2 v \theta_{k}} t^{k+2 v \theta_{k}}}{\left[k+2 v \theta_{k}\right]_{q_{n}} !}\left(1+\frac{1}{\delta}\left(q_{n}^{k+2 v \theta_{k}} t-x\right)^{2}\right) \mathrm{d}_{q_{n}} t\right]^{\frac{1}{2}} \\
&\left.\times\left(\mathcal{P}_{n, q_{n}}^{*}(1 ; x)\right)^{\frac{1}{2}}\right\} \omega(f ; \delta) \\
&=\left\{1+\frac{1}{\delta}\left(\mathcal{P}_{n, q_{n}}^{*}\left(q_{n}^{k+2 v \theta_{k}} t-x\right)^{2} ; x\right)^{\frac{1}{2}}\right\} \omega(f ; \delta),
\end{aligned}
$$

where $\mathcal{P}_{n, q_{n}}^{*}\left(\left(q_{n}^{k+2 v \theta_{k}} t-x\right)^{2} ; x\right) \leq \mathcal{P}_{n, q_{n}}^{*}\left((t-x)^{2} ; x\right)$. And if we now choose $\delta=\delta_{n}=\sqrt{\frac{1}{\left[n q_{n}\right.}}$, then we get our result. 
Corollary 4.2 For $\delta_{n}=\mathcal{P}_{n, q_{n}}^{*}\left(\left(q_{n}^{k+2 v \theta_{k}} t-x\right)^{2} ; x\right)$, we have

$$
\left|\mathcal{P}_{n, q_{n}}^{*}(f ; x)-f(x)\right| \leq 2 \omega\left(f ; \delta_{n}\right)
$$

\section{Rate of convergence}

Now we give the rate of convergence of the operators $\mathcal{P}_{n, q}^{*}(f ; x)$ in terms of the elements of the usual Lipschitz class $\operatorname{Lip}_{M}(v)$.

Let $f \in C[0, \infty), M>0$ and $0<v \leq 1$. The class $\operatorname{Lip}_{M}(v)$ is defined as

$$
\operatorname{Lip}_{M}(v)=\left\{f:\left|f\left(\varsigma_{1}\right)-f\left(\varsigma_{2}\right)\right| \leq M\left|\varsigma_{1}-\varsigma_{2}\right|^{\nu} ;\left(\varsigma_{1}, \varsigma_{2} \in[0, \infty)\right)\right\}
$$

Theorem 5.1 Let $q=q_{n}$ be such that $q_{n} \in(0,1)$ and (3.1) holds. Then, for each $f \in \operatorname{Lip}_{M}(v)$ with $M>0,0<v \leq 1$, we have

$$
\left|\mathcal{P}_{n, q_{n}}^{*}(f ; x)-f(x)\right| \leq M\left(\frac{\left(1+q_{n}\right)}{q_{n}{ }^{3}[n]_{q_{n}}^{2}}+\frac{1}{q_{n}{ }^{2}[n]_{q_{n}}}\left(1+{q_{n}}^{2}[1+2 v]_{q_{n}}\right) x\right)^{\frac{v}{2}}
$$

Proof We prove it by using (5.1) and Hölder's inequality. Indeed,

$$
\begin{aligned}
\left|\mathcal{P}_{n, q_{n}}^{*}(f ; x)-f(x)\right| & \leq\left|\mathcal{P}_{n, q_{n}}^{*}(f(t)-f(x) ; x)\right| \\
& \leq \mathcal{P}_{n, q_{n}}^{*}(|f(t)-f(x)| ; x) \\
& \leq M \mathcal{P}_{n, q_{n}}^{*}\left(\left|q_{n}^{k+2 v \theta_{k}} t-x\right|^{v} ; x\right) .
\end{aligned}
$$

Therefore,

$$
\begin{aligned}
\left|\mathcal{P}_{n, q_{n}}^{*}(f ; x)-f(x)\right| & \\
\leq & M \frac{[n]_{q_{n}}}{e_{v, q_{n}}\left([n]_{q_{n}} x\right)} \sum_{k=0}^{\infty} Q_{n, q_{n}}^{v}(x) \int_{0}^{\infty / 1-q_{n}} \frac{e_{v, q_{n}}\left(-[n]_{q_{n}} t\right)[n]_{q_{n}}^{k+2 v \theta_{k}} t^{k+2 v \theta_{k}}}{\left[k+2 v \theta_{k}\right]_{q_{n}} !} \\
& \times\left|q_{n}^{k+2 v \theta_{k}} t-x\right| \mathrm{d}_{q_{n}} t \\
\leq & M \frac{[n]_{q_{n}}}{e_{v, q_{n}}\left([n]_{q_{n}} x\right)} \sum_{k=0}^{\infty}\left(Q_{n, q_{n}}^{v}(x)\right)^{\frac{2-v}{2}}\left(Q_{n, q_{n}}^{v}(x)\right)^{\frac{v}{2}} \\
& \times \int_{0}^{\infty / 1-q_{n}} \frac{e_{q_{n}}\left(-[n]_{q_{n}} t\right)[n]_{q_{n}}^{k+2 v \theta_{k}} t^{k+2 v \theta_{k}}}{\left[k+2 v \theta_{k}\right]_{q_{n}} !} \mid q_{n}^{k+2 v \theta_{k} t-x \mid \mathrm{d}_{q_{n}} t} \\
\leq & M\left(\frac{[n]_{q_{n}}}{e_{v, q_{n}}\left([n]_{q_{n}} x\right)} \sum_{k=0}^{\infty} Q_{n, q_{n}}^{v}(x) \int_{0}^{\infty / 1-q_{n}} \frac{e_{q_{n}}\left(-[n]_{q_{n}} t\right)[n]_{q_{n}}^{k+2 v \theta_{k}} t^{k+2 v \theta_{k}}}{\left[k+2 v \theta_{k}\right]_{q_{n}} !} \mathrm{d}_{q_{n}} t\right)^{\frac{2-v}{2}} \\
& \times\left(\frac{[n]_{q_{n}}}{e_{v, q_{n}}\left([n]_{q_{n}} x\right)} \sum_{k=0}^{\infty} Q_{n, q_{n}}^{v}(x) \int_{0}^{\infty / 1-q_{n}} \frac{e_{q_{n}}\left(-[n]_{q_{n}} t\right)[n]_{q_{n}}^{k+2 v \theta_{k}} t^{k+2 v \theta_{k}}}{\left[k+2 v \theta_{k}\right]_{q_{n}} !}\right. \\
= & \left.M\left(\mathcal{P}_{n, q_{n}}^{*}\left(q_{n}^{k+2 v \theta_{k}} t-x\right)^{2} ; x\right)^{\frac{v}{2}} \cdot q_{n}^{k+2 v \theta_{k} t-\left.x\right|^{2}} \mathrm{~d}_{q_{n}} t\right)^{\frac{v}{2}} \\
\quad &
\end{aligned}
$$

This completes the proof. 
Let $C_{B}[0, \infty)$ denote the space of all bounded and continuous functions defined on $\mathbb{R}^{+}=$ $[0, \infty)$ and

$$
C_{B}^{2}\left(\mathbb{R}^{+}\right)=\left\{\psi \in C_{B}\left(\mathbb{R}^{+}\right): \psi^{\prime}, \psi^{\prime \prime} \in C_{B}\left(\mathbb{R}^{+}\right)\right\}
$$

with the norm

$$
\|\psi\|_{C_{B}^{2}\left(\mathbb{R}^{+}\right)}=\|\psi\|_{C_{B}\left(\mathbb{R}^{+}\right)}+\left\|\psi^{\prime}\right\|_{C_{B}\left(\mathbb{R}^{+}\right)}+\left\|\psi^{\prime \prime}\right\|_{C_{B}\left(\mathbb{R}^{+}\right)},
$$

also set

$$
\|\psi\|_{C_{B}\left(\mathbb{R}^{+}\right)}=\sup _{x \in \mathbb{R}^{+}}|\psi(x)| .
$$

Theorem 5.2 Let $\mathcal{P}_{n, q}^{*}(\cdot ; \cdot)$ be the operators defined by (1.7). Then, for $q=q_{n}$ such that $q_{n} \in(0,1)$ and any $\psi \in C_{B}^{2}\left(\mathbb{R}^{+}\right)$,

$$
\left|\mathcal{P}_{n, q_{n}}^{*}(\psi ; x)-\psi(x)\right| \leq\left(\Delta_{n, q_{n}}+\Phi_{n, q_{n}}(x)\right)\|\psi\|_{C_{B}^{2}\left(\mathbb{R}^{+}\right)},
$$

where $\Delta_{n, q_{n}}=\frac{1}{q_{n}[n] q_{n}}+\frac{1}{2 q_{n}^{2}[n] q_{n}^{2}}\left(1+\frac{1}{q_{n}}\right)$ and $\Phi_{n, q_{n}}(x)=\frac{1}{2 q_{n}^{2}[n] q_{n}}\left(1+q_{n}^{2}[1+2 v]_{q_{n}}\right) x$.

Proof Let $\psi \in C_{B}^{2}\left(\mathbb{R}^{+}\right)$. Then, by using the generalized mean value theorem in the Taylor series expansion, we have

$$
\psi(t)=\psi(x)+\psi^{\prime}(x)(t-x)+\psi^{\prime \prime}(\varphi) \frac{(t-x)^{2}}{2}, \quad \varphi \in(x, t) .
$$

By applying the linearity property of $\mathcal{P}_{n, q_{n}}^{*}$, we have

$$
\mathcal{P}_{n, q_{n}}^{*}(\psi ; x)-\psi(x)=\psi^{\prime}(x) \mathcal{P}_{n, q_{n}}^{*}((t-x) ; x)+\frac{\psi^{\prime \prime}(\varphi)}{2} \mathcal{P}_{n, q_{n}}^{*}\left((t-x)^{2} ; x\right),
$$

which implies that

$$
\begin{aligned}
& \left|\mathcal{P}_{n, q_{n}}^{*}(\psi ; x)-\psi(x)\right| \\
& \quad \leq\left(\frac{1}{q_{n}[n]_{q_{n}}}\right)\left\|\psi^{\prime}\right\|_{C_{B}\left(\mathbb{R}^{+}\right)} \\
& \quad+\left\{\frac{\left(1+q_{n}\right)}{q_{n}^{3}[n]_{q_{n}}^{2}}+\frac{1}{q_{n}^{2}[n]_{q_{n}}}\left(1+q_{n}{ }^{2}[1+2 v]_{q_{n}}\right) x\right\} \frac{\left\|\psi^{\prime \prime}\right\|_{C_{B}\left(\mathbb{R}^{+}\right)}}{2} .
\end{aligned}
$$

From (5.3) we have $\left\|\psi^{\prime}\right\|_{C_{B}\left(\mathbb{R}^{+}\right)} \leq\|\psi\|_{C_{B}^{2}\left(\mathbb{R}^{+}\right)}$and $\left\|\psi^{\prime \prime}\right\|_{C_{B}\left(\mathbb{R}^{+}\right)} \leq\|\psi\|_{C_{B}^{2}\left(\mathbb{R}^{+}\right)}$, as well as

$$
\begin{aligned}
& \left|\mathcal{P}_{n, q_{n}}^{*}(\psi ; x)-\psi(x)\right| \\
& \leq\left(\frac{1}{q_{n}[n]_{q_{n}}}\right)\|\psi\|_{C_{B}^{2}\left(\mathbb{R}^{+}\right)} \\
& \quad+\left\{\frac{\left(1+q_{n}\right)}{q_{n}^{3}[n]_{q_{n}}^{2}}+\frac{1}{q_{n}^{2}[n]_{q_{n}}}\left(1+q_{n}^{2}[1+2 v]_{q_{n}}\right) x\right\} \frac{\|\psi\|_{C_{B}^{2}\left(\mathbb{R}^{+}\right)}}{2} .
\end{aligned}
$$

This completes the proof. 


\section{Direct theorem}

In 1968, J. Peetre [22] introduced a functional known as Peetre's K-functional, which is defined by

$$
K_{2}(f ; \delta)=\inf \left\{\left(\|f-\psi\|_{C_{B}\left(\mathbb{R}^{+}\right)}+\delta\|\psi\|_{C_{B}^{2}\left(\mathbb{R}^{+}\right)}\right): \psi \in C_{B}^{2}\left(\mathbb{R}^{+}\right)\right\}
$$

There exits a positive constant $C>0$ such that $K_{2}(f, \delta) \leq C \omega_{2}\left(f, \delta^{\frac{1}{2}}\right), \delta>0$, where the second-order modulus of continuity is given by

$$
\omega_{2}(f ; \delta)=\sup _{0<h<\delta} \sup _{x \in \mathbb{R}^{+}}|f(x+2 h)-2 f(x+h)+f(x)| .
$$

Theorem 6.1 For $f \in C_{B}\left(\mathbb{R}^{+}\right), x \in[0, \infty)$ and $q=q_{n}$ satisfying (3.1), we have

$$
\begin{aligned}
& \left|\mathcal{P}_{n, q_{n}}^{*}(f ; x)-f(x)\right| \\
& \quad \leq 2 \mathcal{D}\left\{\omega_{2}\left(f ; \sqrt{\frac{\Delta_{n, q_{n}}+\Phi_{n, q_{n}}(x)}{2}}\right)+\min \left(1, \frac{\Delta_{n, q_{n}}+\Phi_{n, q_{n}}(x)}{2}\right)\|f\|_{C_{B}\left(\mathbb{R}^{+}\right)}\right\},
\end{aligned}
$$

where $\mathcal{D}$ is a positive constant.

Proof We prove this by using Theorem 5.2. Let $\psi \in C_{B}\left(\mathbb{R}^{+}\right)$, then

$$
\begin{aligned}
\left|\mathcal{P}_{n, q_{n}}^{*}(f ; x)-f(x)\right| & \leq\left|\mathcal{P}_{n, q_{n}}^{*}(f-\psi ; x)\right|+\left|\mathcal{P}_{n, q_{n}}^{*}(\psi ; x)-\psi(x)\right|+|f(x)-\psi(x)| \\
& \leq 2\|f-\psi\|_{C_{B}\left(\mathbb{R}^{+}\right)}+\left(\Delta_{n, q_{n}}+\Phi_{n, q_{n}}(x)\right)\|\psi\|_{C_{B}^{2}\left(\mathbb{R}^{+}\right)} \\
& =2\left(\|f-\psi\|_{C_{B}\left(\mathbb{R}^{+}\right)}+\frac{\Delta_{n, q_{n}}+\Phi_{n, q_{n}}(x)}{2}\|\psi\|_{C_{B}^{2}\left(\mathbb{R}^{+}\right)}\right) .
\end{aligned}
$$

By taking the infimum over all $\psi \in C_{B}^{2}\left(\mathbb{R}^{+}\right)$and using (6.1), we get

$$
\left|\mathcal{P}_{n, q_{n}}^{*}(f ; x)-f(x)\right| \leq 2 K_{2}\left(f ; \frac{\Delta_{n, q_{n}}+\Phi_{n, q_{n}}(x)}{2}\right) .
$$

Now, for an absolute constant $\mathcal{D}>0$ provided in [7], we use the relation

$$
K_{2}(f ; \delta) \leq \mathcal{D}\left\{\omega_{2}(f ; \sqrt{\delta})+\min (1, \delta)\|f\|\right\}
$$

This completes the proof.

Atakut and Ispir [5] introduced the weighted modulus of continuity defined as

$$
\Omega(f ; \delta)=\sup _{x \in[0, \infty),|h| \leq \delta} \frac{|f(x+h)-f(x)|}{\left(1+h^{2}\right)\left(1+x^{2}\right)},
$$

for an arbitrary $f \in Q_{\sigma}^{k}\left(\mathbb{R}^{+}\right)$. The two main properties of this modulus of continuity are $\lim _{\delta \rightarrow 0} \Omega(f ; \delta)=0$ and

$$
|f(t)-f(x)| \leq 2\left(1+\frac{|t-x|}{\delta}\right)\left(1+\delta^{2}\right)\left(1+x^{2}\right)\left(1+(t-x)^{2}\right) \Omega(f ; \delta),
$$

where $t, x \in[0, \infty)$. 
Theorem 6.2 Let $q=q_{n}$ be numbers such that $q_{n} \in(0,1)$ as $n \rightarrow \infty$. Then, for every $f \in$ $Q_{\sigma}^{k}\left(\mathbb{R}^{+}\right)$

$$
\sup _{x \in\left[0, \chi_{v, q_{n}}(n)\right)} \frac{\left|\mathcal{P}_{n, q_{n}}^{*}(f ; x)-f(x)\right|}{1+x^{2}} \leq \mathcal{C}\left(1+\chi_{v, q_{n}}(n)\right) \Omega\left(f ; \sqrt{\chi_{v, q_{n}}}\right),
$$

where the positive constant $\mathcal{C}=1+\mathcal{C}_{1}+4 \mathcal{C}_{2}$ and

$$
\chi_{v, q_{n}}(n)=\max \left\{\frac{\left(1+q_{n}\right)}{{q_{n}{ }^{3}[n]_{q_{n}}^{2}}^{2}}, \frac{1}{{q_{n}{ }^{2}[n]_{q_{n}}}_{n_{n}}}\left(1+q_{n}^{2}[1+2 v]_{q_{n}}\right)\right\} .
$$

Proof We use (6.3)-(6.4) and the Cauchy-Schwarz inequality. Thus we have

$$
\begin{aligned}
& \left|\mathcal{P}_{n, q_{n}}^{*}(f ; x)-f(x)\right| \\
& \quad \leq 2\left(1+\delta^{2}\right)\left(1+x^{2}\right) \Omega(f ; \delta) \\
& \quad \times\left(1+\mathcal{P}_{n, q_{n}}^{*}\left((t-x)^{2} ; x\right)+\mathcal{P}_{n, q_{n}}^{*}\left(\left(1+(t-x)^{2}\right) \frac{|t-x|}{\delta} ; x\right)\right)
\end{aligned}
$$

and

$$
\begin{aligned}
& \mathcal{P}_{n, q_{n}}^{*}\left(\left(1+(t-x)^{2}\right) \frac{|t-x|}{\delta} ; x\right) \\
& \quad \leq 2\left(\mathcal{P}_{n, q_{n}}^{*}\left(1+(t-x)^{4} ; x\right)\right)^{\frac{1}{2}}\left(\mathcal{P}_{n, q_{n}}^{*}\left(\frac{(t-x)^{2}}{\delta^{2}} ; x\right)\right)^{\frac{1}{2}} .
\end{aligned}
$$

From Lemma 2.2, we easily see that

$$
\mathcal{P}_{n, q_{n}}^{*}\left((t-x)^{2} ; x\right) \leq \chi_{v, q_{n}}(n)(1+x),
$$

where

$$
\chi_{v, q_{n}}(n)=\max \left\{\frac{\left(1+q_{n}\right)}{q_{n}{ }^{3}[n]_{q_{n}}^{2}}, \frac{1}{q_{n}^{2}[n]_{q_{n}}}\left(1+q_{n}^{2}[1+2 v]_{q_{n}}\right)\right\} .
$$

Now there exits a constant $\mathcal{C}_{1}>0$ such that

$$
\mathcal{P}_{n, q_{n}}^{*}\left((t-x)^{2} ; x\right) \leq \mathcal{C}_{1}(1+x) .
$$

We easily conclude that

$$
\begin{aligned}
\mathcal{P}_{n, q_{n}}^{*}\left((t-x)^{4} ; x\right) \leq & \frac{24}{q_{n}^{10}[n]_{q_{n}}^{4}} \\
& +\frac{1}{q_{n}^{9}[n]_{q_{n}}^{3}}\left(26+35[1+2 v]_{q_{n}}+10[1+2 v]_{q_{n}}^{2}+[1+2 v]_{q_{n}}^{3}\right) x \\
& +\frac{1}{q_{n}^{8}[n]_{q_{n}}^{2}}\left(3-14[1+2 v]_{q_{n}}+3[1+2 v]_{q_{n}}^{2}\right) x^{2} \\
& +\frac{1}{q_{n}^{5}[n]_{q_{n}}}\left(-10-6[1+2 v]_{q_{n}}\right) x^{3}+8 x^{4} \\
\leq & \xi_{v, q_{n}}(n)\left(1+x+x^{2}+x^{3}+x^{4}\right),
\end{aligned}
$$


where

$$
\begin{aligned}
\xi_{v, q_{n}}(n)= & \max \left\{\frac{24}{q_{n}^{10}[n]_{q_{n}}^{4}}, \frac{1}{q_{n}^{9}[n]_{q_{n}}^{3}}\left(26+35[1+2 v]_{q_{n}}+10[1+2 v]_{q_{n}}^{2}+[1+2 v]_{q_{n}}^{3}\right),\right. \\
& \left.\frac{1}{q_{n}^{8}[n]_{q_{n}}^{2}}\left(3-14[1+2 v]_{q_{n}}+3[1+2 v]_{q_{n}}^{2}\right), \frac{1}{q_{n}^{5}[n]_{q_{n}}}\left(-10-6[1+2 v]_{q_{n}}\right), 8\right\} .
\end{aligned}
$$

Since, $\lim _{n \rightarrow \infty} \frac{1}{[n]_{q_{n}}^{i}}=0$ for all $i=1,2,3,4$ and $\lim _{n \rightarrow \infty} q_{n}=1$, for a constant $\mathcal{C}_{1}>0$, we have

$$
\left(\mathcal{P}_{n, q_{n}}^{*}\left(1+(t-x)^{4} ; x\right)\right)^{\frac{1}{2}} \leq \mathcal{C}_{2}\left(2+x+x^{2}+x^{3}+x^{4}\right)^{\frac{1}{2}} .
$$

In the view of (6.7), we easily see that

$$
\left(\mathcal{P}_{n, q_{n}}^{*}\left(\frac{(t-x)^{2}}{\delta^{2}} ; x\right)\right)^{\frac{1}{2}} \leq \frac{1}{\delta}\left(\chi_{v, q_{n}}(n)\right)^{\frac{1}{2}}(1+x)^{\frac{1}{2}}
$$

Finally, in the light of equation (6.5) by combining (6.6)-(6.10), if we choose $\delta=$ $\sqrt{\chi_{v, q_{n}}(n)}$ and take the supremum over $x \in\left[0, \chi_{v, q_{n}}(n)\right)$, we get the desired result.

Funding

The second author would like to thank Prince Sultan University for funding this work through research group "Nonlinear Analysis Methods in Applied Mathematics (NAMAM)" group number RG-DES-2017-01-17.

\section{Competing interests}

The authors declare that they have no competing interests.

\section{Authors' contributions}

The authors contributed equally and significantly in writing this paper. All authors read and approved the final manuscript.

\section{Author details}

${ }^{1}$ Department of Computer Science (SEST), Jamia Hamdard, New Delhi, India. ${ }^{2}$ Department of Mathematics and General Sciences, Prince Sultan University, Riyadh, Saudi Arabia. ${ }^{3}$ Department of Mathematics, Aligarh Muslim University, Aligarh, India.

\section{Publisher's Note}

Springer Nature remains neutral with regard to jurisdictional claims in published maps and institutional affiliations.

Received: 17 December 2018 Accepted: 4 June 2019 Published online: 21 June 2019

\section{References}

1. Acar, T:: Quantitative $q$-Voronovskaya and $q$-Grüss-Voronovskaya-type results for $q$-Szász operators. Georgian Math. J. 23, 459-468 (2016)

2. Acar, T., Aral, A.: On pointwise convergence of $q$-Bernstein operators and their $q$-derivatives. Numer. Funct. Anal. Optim. 36, 287-304 (2015)

3. Alotaibi, A., Nasiruzzaman, M., Mursaleen, M.: A Dunkl type generalization of Szász operators via post-quantum calculus. J. Inequal. Appl. 2018, Article ID 287 (2018)

4. Altomare, F.: Korovkin type theorems and approximation by positive linear operators. Surv. Approx. Theory 5, 92-164 (2010)

5. Atakut, C., Ispir, N.: Approximation by modified Szász-Mirakjan operators on weighted spaces. Proc. Indian Acad. Sci. Math. Sci. 112, 571-578 (2002)

6. Cheikh, B., Gaied, Y., Zaghouani, M.: A q-Dunkl-classical q-Hermite type polynomials. Georgian Math. J. 21, 125-137 (2014)

7. Ciupa, A.: A class of integral Favard-Szász type operators. Stud. Univ. Babeş-Bolyai, Math. 40, 39-47 (1995)

8. De Sole, A., Kac, V.G.: On integral representation of q-gamma and q-beta functions. Atti Accad. Naz. Lincei, Rend. Lincei, Mat. Appl. 16, 11-29 (2005)

9. Gairola, A.R., Deepmala, Mishra, L.N.: Rate of approximation by finite iterates of $q$-Durrmeyer operators. Proc. Natl. Acad. Sci. India Sect. A Phys. Sci. 86, 229-234 (2016)

10. Gairola, A.R., Deepmala, Mishra, L.N.: On the q-derivatives of a certain linear positive operators. Iran. J. Sci. Technol., Trans. A, Sci. 42, 1409-1417 (2018) 
11. İçöz, G., Çekim, B.: Dunkl generalization of Szász operators via q-calculus. J. Inequal. Appl. 2015, Article ID 284 (2015)

12. Içöz, G., Çekim, B.: Stancu type generalization of Dunkl analogue of Szász-Kantorovich operators. Math. Methods Appl. Sci. 39, 1803-1810 (2016)

13. Jackson, F.H.: On q-definite integrals. Q. J. Pure Appl. Math. 15, 193-203 (1910)

14. Lupaş, A.: A q-analogue of the Bernstein operator. Univ. Cluj-Napoca Semin. Numer. Stat. Calc. 9, 85-92 (1987)

15. May, C.P.: On Phillips operators. J. Approx. Theory 20, 315-322 (1977)

16. Milovanovic, G.V., Mursaleen, M., Nasiruzzaman, M.: Modified Stancu type Dunkl generalization of Szász-Kantorovich operators. Rev. R. Acad. Cienc. Exactas Fís. Nat., Ser. A Mat. 112, 135-151 (2018)

17. Mishra, V.N., Khatri, K., Mishra, L.N.: Statistical approximation by Kantorovich type discrete $q$-beta operators. Adv. Differ. Equ. 2013, Article ID 345 (2013)

18. Mishra, V.N., Khatri, K., Mishra, L.N.: Some approximation properties of q-Baskakov-Beta-Stancu type operators. J. Calc. Var. 2013, Article ID 814824 (2013)

19. Mishra, V.N., Pandey, S., Khan, I.A.: On a modification of Dunkl generalization of Szász operators via q-calculus. Eur. J. Pure Appl. Math. 10, 1067-1077 (2017)

20. Mursaleen, M., Nasiruzzaman, M., Alotaibi, A.: On modified Dunkl generalization of Szász operators via q-calculus. J. Inequal. Appl. 2017, Article ID 38 (2017)

21. Nasiruzzaman, M., Rao, N.: A generalized Dunkl type modifications of Phillips operators. J. Inequal. Appl. 2018, Article ID 323 (2018)

22. Peetre, J.: A Theory of Interpolation of Normed Spaces. Notas de mathematica, vol. 39. Instituto de Mathemática Pura e Applicada, Conselho Nacional de Pesquidas Rio de Janeiro (1968)

23. Rao, N., Wafi, A., Acu, A.M.: q-Szász-Durrmeyer type operators based on Dunkl analogue. Complex Anal. Oper. Theory (2019). https://doi.org/10.1007/s11785-018-0816-3

24. Rosenblum, M.: Generalized Hermite polynomials and the Bose-like oscillator calculus. Oper. Theory, Adv. Appl. 73, 369-396 (1994)

25. Srivastava, H.M., Mursaleen, M., Alotaibil, A., Nasiruzzaman, M., Al-Abied, A.: Some approximation results involving the q-Szasz-Mirakjan-Kantorovich type operators via Dunkl's generalization. Math. Methods Appl. Sci. 40, 5437-5452 (2017)

26. Sucu, S.: Dunkl analogue of Szász operators. Appl. Math. Comput. 244, 42-48 (2014)

27. Szász, O.: Generalization of S. Bernstein's polynomials to the infinite interval. J. Res. Natl. Bur. Stand. 45, $239-245$ (1950)

28. Ulusoy, G., Acar, T:: q-Voronovskaya type theorems for q-Baskakov operators. Math. Methods Appl. Sci. 39, 3391-3401 (2016)

\section{Submit your manuscript to a SpringerOpen ${ }^{\circ}$ journal and benefit from:}

- Convenient online submission

- Rigorous peer review

- Open access: articles freely available online

- High visibility within the field

- Retaining the copyright to your article

Submit your next manuscript at $>$ springeropen.com 